propuestos por los autores y practicar de forma autónoma añade aún más interés a la propuesta.

\section{Optimización de las competencias del traductor e intérprete: Nuevas tecnologías - procesos cognitivos - estrategias}

\section{EnCarnación Postigo Pinazo (ED.)}

Berlín, Frank \& Timme, 2018, 184 págs.

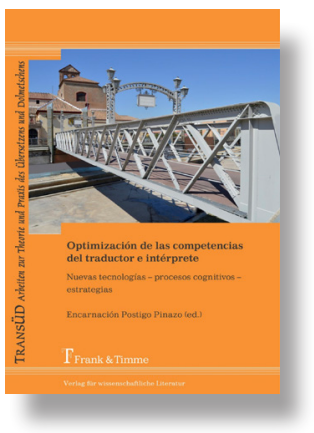

Este libro consta de nueve capítulos que cubren el uso de las nuevas tecnologías en traducción e interpretación, la formación y los aspectos profesionales y éticos de la labor de los traductores e intérpretes en varios ámbitos, incluidas zonas de conflicto, la traducción inversa y las bibliotecas electrónicas. El volumen ha sido realizado en el marco del proyecto de innovación educativa PIE 15-41 (2015-2017) de la Universidad de Málaga con las Universidades de Portsmouth (Reino Unido) y la Universidad de Gante (Bélgica) denominado: Recursos TIC y estrategias para una optimización proactiva del aprendizaje de lenguas extranjeras y de las competencias para la traducción e interpretación: experiencia pedagógica compartida con dos universidades europeas socias. Todos los trabajos son resultado de la colaboración de investigadores de las tres instituciones mencionadas que, a su vez, mantienen estrechas relaciones de cooperación dentro del programa de intercambio Erasmus.

Las aportaciones subrayan la importancia de las nuevas tecnologías en el campo de la traducción e interpretación y una adecuada y actualizada formación de los traductores, mediante metodologías efectivas y de acuerdo con los nuevos estándares profesionales. Además, examinan los códigos éticos, las condiciones laborales y los efectos del estrés en la labor de traductores e intérpretes, y presentan una importante contribución de las humanidades digitales al conocimiento y estudio del patrimonio traducido en España e Hispanoamérica.

El volumen consta de una introducción, realizada por Nalini Boodhoo (University of East Anglia), quien destaca dos bloques en el libro: uno dedicado a la interpretación y otro a la traducción. El libro empieza con el capítulo denominado «LATS (Language Assessment Training System) - una herramienta TIC para la enseñanza activa de competencias en diagnóstico clínico en el ámbito de los problemas del lenguaje», que precede a dichos bloques, y corre a cargo de María Calleja Reina, José Miguel Rodríguez Santos y María Luisa Luque Liñán. Los investigadores abogan por la formación basada en el aprendizaje activo, y presentan una herramienta creada con el objetivo de entrenar los estudiantes del Grado de Logopedia en razonamiento clínico diagnóstico, combinando la enseñanza presencial y la presentación por ordenador con el objetivo de resolver casos de la dificultad de lenguaje y poder repetir la experiencia. La herramienta puede resultar muy útil tanto para los estudiantes como para los docentes universitarios.

A continuación, el capítulo de Hildegard Vermeiren, «Interpretación y tecnologías para la comunicación: propuestas concretas para formadores», tal y como indica el título, sobre todo se dirige a los docentes y defiende la postura a favor del uso y la formación de los intérpretes en las TICs. La autora reconoce que el empleo de las nuevas tecnologías en el ámbito de la interpre- 
602 tación, como son los bancos de datos terminológicos, programas del reconocimiento del habla, la traducción automática y otras herramientas, puede provocar sentimientos ambivalentes entre los profesionales, pero insiste en que los centros de formación deben ayudar a los alumnos y alumnas a adquirir los conocimientos necesarios en TICs y saber manejarlos en su futura labor profesional. En ese sentido, aborda la metodología usada para fomentar el uso de las tecnologías más avanzadas y ciertas técnicas de interpretación menos conocidas como shadowing, interpretación a vista, interpretación móvil, interpretación a distancia o videoconferencias, etc., en la asignatura «Técnica y Tecnología» en la Universidad de Gante, ofreciendo a la vez una serie de actividades prácticas, basadas en su experiencia como intérprete, por un lado, y docente por otro.

En el capítulo denominado «Análisis de las causas de estrés y ansiedad anticipativa en la interpretación simultánea desde una perspectiva interdisciplinar: estudio piloto comparativo entre profesionales y estudiantes», realizado por Paloma Ojeda Fernández y Encarnación Postigo Pinazo de la Universidad de Málaga, se ahonda en el tema del estrés y sus posibles causas a la hora de realizar la labor de la interpretación simultánea. Se presentan los resultados de un estudio piloto de carácter observacional, comparativo, cuantitativo y empírico, realizado a través de los cuestionarios que se han entregado a los profesionales de la interpretación simultánea y a los estudiantes de esta asignatura.

Las autoras también comentan unas valiosas fuentes de referencia disponibles en la actualidad, concluyendo que se echan de menos más investigaciones sobre esta temática. A continuación, señalan posibles factores que podrían producir el estrés y analizan las respuestas de los entrevistados en relación con estas situaciones, señalando una mayor falta de confianza en el caso de los es- tudiantes que en el caso de los profesionales, así como otras diferencias significativas e interesantes entre las respuestas de estos dos grupos.

El siguiente capítulo, «Ansiedad ante un examen final oral en estudiantes universitarios de Traducción e Interpretación. ¿La ansiedad influye en la nota?», también versa sobre el tema del estrés. En él, Ana Masedo Gutiérrez analiza la ansiedad experimentada por parte de los estudiantes en el momento de la evaluación oral y la ansiedad como un rasgo relacionado con su forma de ser. Se realiza un estudio empírico con la participación de los estudiantes del Grado en Traducción e Interpretación de la Universidad de Málaga para averiguar si sienten ansiedad durante las pruebas orales del fin de curso, y si existe una estrecha relación entre la ansiedad que han experimentado y su nota en el examen.

El siguiente artículo, «Códigos éticos: formación para intérpretes», inicia la sección del libro sobre el código ético y la labor o la formación de los intérpretes. La autora, Laura Parrilla Gómez (Universidad Pablo de Olavide), señala una falta de dedicación de tiempo en la formación de los intérpretes a los aspectos como la ética profesional en comparación con algunos otros campos especializados, y describe el panorama actual de la existencia de los estándares de actuación y los códigos éticos establecidos por las asociaciones en el campo de la interpretación a nivel nacional e internacional, sobre todo europeo. Igualmente, se señala la importancia de tales normas por el bien de las dos partes que reciben el servicio, pero también de los intérpretes que deben conocer y tener acceso a un código deontológico. El estudio que sigue se basa en una investigación previa, de 2015, realizada por Postigo Pinazo sobre la presencia de la ética profesional en la formación de intérpretes, y analiza los encuentros reales entre los intérpretes voluntarios y los pacientes de un hospital de la Costa del Sol, cen- 
trándose en los aspectos de la ética profesional. Una de las conclusiones de dicho estudio es la importancia de contar con los intérpretes profesionales, competentes y preparados, que conozcan los estándares profesionales y las delicadas situaciones con las que se puede enfrentar un profesional de la interpretación médica.

El siguiente apartado, «Aspectos éticos y profesionales en el desempeño de la labor de traductores e intérpretes en zonas de conflicto: semblanzas y casos», que corre a cargo de Mohamed H. Al-Azawi y Marcos Rodríguez Espinosa, profundiza en el tema de los aspectos profesionales y éticos de la interpretación. Los autores recopilan la información de la prensa y bibliografía especializada acerca de los intérpretes nativos o residentes de zonas de conflicto, y ponen en evidencia sus precarias condiciones laborales a pesar del peligro al que se enfrentan y las catastróficas consecuencias que algunos llegan a sufrir por colaborar con las fuerzas extranjeras, como ocurre a menudo en las zonas de Irán o Afganistán. Aparte de enfatizar los principios éticos de la profesión, e insistir en el papel de intérprete como un intermediador cultural que, cuando trabaja en este tipo de situaciones, necesita guiarse también por su criterio personal ( $\mathrm{p}$. 112 ), recuerdan que los derechos de los intérpretes deberían estar garantizados por escrito.

En el artículo, se relatan varios casos de intérpretes que han sufrido daños graves, han resultado heridos o han perdido la vida, con una clara intención de darles la visibilidad a los que ejercen esta importante profesión y reclamar unas mejores condiciones de trabajo y su protección. Se denuncia también el trato injusto que sufren traductores e intérpretes nativos, quienes por falta de experiencia o su origen humilde aceptan contratos que llegan a ser abusivos, así como el peligro en el que viven por no poder acceder a visados o asilos en los países de 603 las fuerzas que han servido.

En el capítulo titulado «El corpus como herramienta auxiliar de la traducción inversa y su aplicación a la mejora de la competencia lingüística de los traductores», Rocío Lavado Puyol se centra en la traducción inversa, una actividad poco investigada en España, que se ejerce en este territorio por falta de traductores nativos a pesar de que algunos profesionales creen que su uso es poco ético. A partir de un estudio basado en las encuestas, se demuestra que la traducción inversa en España es una realidad y algo necesario, y se insiste en la utilidad del corpus en la realización de este tipo de encargos y en la corrección de los errores.

Begoña Rodríguez de Céspedes (Portsmouth University) en su artículo «Mind the gap: Language Service Providers' views on the technological training of professional translators», indaga sobre el impacto de la traducción automática (MT, Machine Translation) y las herramientas de la traducción asistida (Computer Assisted Translation, CAT) en la labor del traductor y su presencia en la formación de los traductores. La autora relata la evolución del proceso de traducción y presenta los resultados de un interesante estudio cualitativo sobre el uso de las herramientas digitales en empresas de traducción en el Reino Unido. Entre otras, el estudio plantea la cuestión de si la responsabilidad de la formación de los traductores en el uso de nuevas tecnologías debería recaer sobre la empresa, el traductor, las asociaciones profesionales o las universidades.

El último capítulo, «Humanidades digitales e historia de la traducción: La aportación de la biblioteca Traducciones y Traductores de Literatura y Ensayo (TTLE) al conocimiento y estudio del patrimonio bibliográfico traducido en España e Hispanoamérica», corre a cargo de Carmen 
604 Acuña-Partal y Juan Jesús Zaro, que presentan la biblioteca virtual TTLE, y resaltan el valor de las Humanidades Digitales como fuente de información sustancial para futuras investigaciones y para la labor docente. Este tipo de datos serían muy dispersos u olvidados sin el trabajo que realizan grupos de investigación con las bibliotecas electrónicas y los bases de datos. La biblioteca electrónica que se describe en el capítulo es fruto del trabajo del grupo de investigación «Traducción y Lenguajes Especializados» (HUM-800), dirigido por el catedrático de la Universidad de Málaga Juan Jesús Zaro, y se compone de las traducciones que han sido recopiladas y editadas en el desarrollo de los Proyectos de Investigación «Archivo digitalizado y edición traductológica de textos literarios y ensayísticos traducidos al español» $\mathrm{y}$ «La traducción como actividad editorial en la Andalucía del siglo xIx: Catálogo y archivo digitalizado», así como del propio Catálogo de traducciones publicadas en Andalucía en el siglo XIX. Estos proyectos de investigación cumplen con sus objetivos de rescatar y editar una selección de textos del patrimonio bibliográfico en español de inmenso valor, reuniendo no solo las traducciones de obras sino también los tratados sobre la traducción. El paso siguiente ha sido la formación del archivo digitalizado de las traducciones y sus ediciones traductológicas, y la difusión de los resultados a través de un portal de Internet, así como su explotación. La importante novedad de esta biblioteca es que las obras fueron editadas según los criterios traductológicos, algo muy valioso para el campo de los Estudios de Traducción, así como para la Historia de la Traducción en España e Hispanoamérica.

Los capítulos del presente volumen, además de presentar los resultados de unas bien fundadas e innovadoras investigaciones en el campo de la traducción e interpretación, aportan los datos y las fuentes de información que resultan realmente ventajosos para los docentes, estudiantes, profesionales e investigadores. Además, se proponen líneas de investigación novedosas que pueden tener continuidad en el futuro.

\section{La traducción de contratos de compra- venta inmobiliaria: un estudio basado en corpus aplicado a España e Irlanda}

\section{Miriam Seghiri y Lorena Arce Romeral}

Berlín, Peter Lang, 2021, 264 págs.

Míriam Pérez-Carrasco

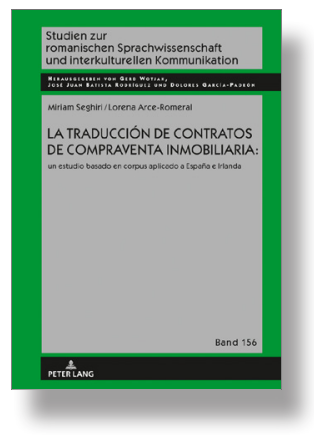

A lo largo de las 264 páginas que conforman este volumen, titulado $L a$ traducción de contratos de compraventa inmobiliaria: un estudio basado en corpus aplicado a España e Irlanda, Seghiri y Arce exponen los resultados de una investigación en la línea de la Lingüística de Corpus, aplicada en concreto a la traducción de textos del ámbito jurídico. Antes de entrar en la reseña del contenido del volumen, cabe subrayar que el trabajo que se recoge en el mismo recibió el premio George Campbell del Aula María Zambrano de Estudios Transatlánticos de la Universidad de Málaga en diciembre de 2019 por ser un contenido original y de gran utilidad para la población residente irlandesa en España, lo cual demuestra, ya de partida, la relevancia de la obra que aquí se reseña.

Seghiri y Arce plantean como hipótesis inicial que «es posible crear herramientas basadas en corpus que sean fáciles y rápidas de construir, reu- 